Excellent Woman Researcher Award of The Electrochemical Society of Japan

\title{
Development of Photofunctional Devices Based on Organic-Inorganic Hybrid Structures
}

\author{
Ayumi ISHII ${ }^{a, b, *, \S ~(D) ~}$
}

${ }^{a}$ Faculty of Life and Environmental Sciences, Teikyo University of Science, 2525 Yatsusawa, Uenohara, Yamanashi 409-0193, Japan

b JST, PRESTO, 4-1-8 Honcho, Kawaguchi, Saitama 332-0012, Japan

\section{*Corresponding author: ayumi-i@ntu.ac.jp}

\section{ABSTRACT}

In this research, organic-inorganic hybrid materials that enable the detection and manipulation of "invisible light" such as weak light, polarized light, and near-infrared (NIR) light are prepared and optoelectronic devices based on these materials are developed. The photoelectric conversion or energy transfer process resulting from light absorption is precisely controlled at the heterointerface of organicinorganic hybrid structures, which enables the highly efficient amplification, conversion, and detection of invisible light under normal temperatures and pressures. Here, novel optical functions and devices based on organic-inorganic hybrid structures and interfaces are presented. For instance, in a hybrid structure in which organic molecules and inorganic semiconductors are chemically bonded, photocurrent was amplified more than 2000-fold at their heterointerface, resulting in highly sensitive photodetection at a low voltage $(<1 \mathrm{~V})$. As a novel device structure for the direct detection of circularly polarized light with high sensitivity, an inorganic crystal thin film with a one-dimensional helical structure was fabricated via interaction with organic chiral molecules. For

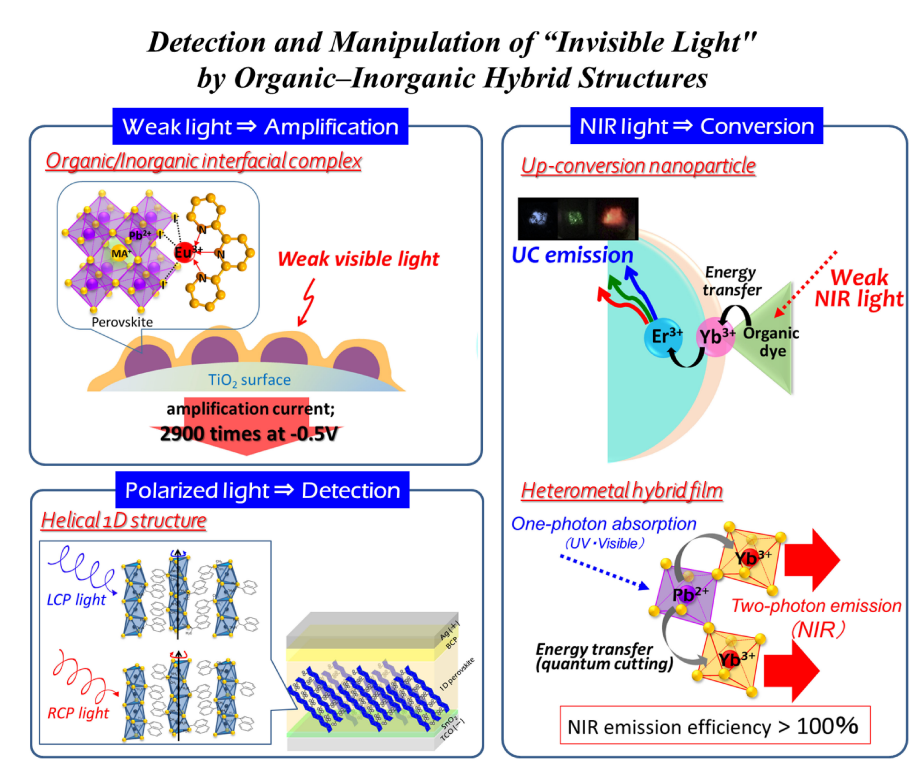
NIR light, dye-sensitized up-conversion nanoparticles that can convert NIR light as weak as sunlight into visible light with high efficiency were developed and incorporated into a perovskite-based visible-light detector. This device detected light in the NIR region through energy conversion from NIR to visible light. And also, NIR light was promoted as ultra-bright luminescence by one-photon absorption two-photon emission (quantum-cutting) process in heterometal hybridized crystal thin films. The light-emitting diode was fabricated and demonstrated $6 \%$ external conversion efficiency of field emission in the NIR region.

(C) The Author(s) 2021. Published by ECSJ. This is an open access article distributed under the terms of the Creative Commons Attribution 4.0 License (CC BY, http://creativecommons.org/licenses/by/4.0/), which permits unrestricted reuse of the work in any medium provided the original work is properly cited. [DOI: 10.5796/electrochemistry.21-00090].

Keywords : Organic-inorganic Hybrid, Photofunctional Materials, Interface, Energy Transfer

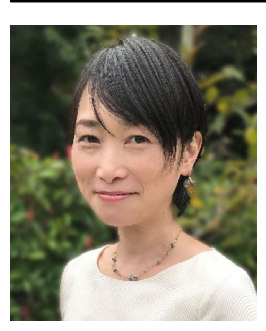

Ayumi Ishii (Associate Professor, Faculty of Life and Environmental Sciences, Teikyo University of Science)

Ayumi Ishii received her Ph.D. in Science from Aoyama Gakuin University in 2008. She worked as a researcher at Advanced Materials Laboratories of SONY Corporation (2008-2011), and then as an assistant professor at Toin University (2011-2014, 2018-2020 as a lecturer) and Aoyama Gakuin University (2014-2018). She currently works as an associate professor from 2020 at Teikyo University of Science. She has been a researcher of JST PRESTO project from 2017. Her research interests are photofunctional materials and devices based on organic-inorganic hybrid structures.

${ }^{\S}$ ECSJ Active Member

A. Ishii (D) orcid.org/0000-0002-8034-3710

\section{Introduction}

Light contains a variety of information and functions, such as intensity, wavelength, polarization, and time (velocity). Elucidating scientific phenomena such as quantum optics and biological functions, as well as enabling the highly efficient utilization of light energy, necessitates the development of new technologies for the accurate detection and manipulation of light. In particular, the development of materials and devices that can accurately detect and effectively use "invisible light" such as weak light, polarized light, and near-infrared (NIR) light is required. However, conventional technologies and materials cannot easily detect weak light under low driving voltages and at normal temperatures and pressures; in addition, polarized light cannot be detected directly. For NIR light, high-brightness and highsensitivity detection are challenging problems.

Research and development in the detection of weak light has focused mainly on visible light. Photomultiplier tubes, ${ }^{1}$ avalanche 
photodiodes (semiconductor photodetectors), ${ }^{2,3}$ superconducting detectors, ${ }^{4}$ and superconducting transition edge sensors ${ }^{5}$ enable the measurement of the intensity (photon number) of weak light at the photon level but only under an applied high voltage (several hundred volts or more) and cryogenic temperatures $(<2 \mathrm{~K})$. Such high-precision optical measurements have led to a wide range of scientific and technological advances, including spectroscopic analysis, astronomical observations, bioanalyses, environmental measurements, and communication technologies. ${ }^{6-9}$ Although existing photodetectors can detect the intensity of light (number of photons), they cannot directly detect light information such as wavelength and polarization. Therefore, obtaining information related to wavelength and polarization necessitates the spatial separation of light information by, for example, combining it with diffraction gratings or filters such as polarizers. This approach not only reduces the detection sensitivity but also removes some of the information from light.

NIR light is light in the wavelength region $780-2500 \mathrm{~nm}$, which is invisible to the human eye. The properties of this invisible NIR light are routinely used in a wide range of fields such as infrared cameras (night-vision cameras), infrared communication (wireless communication), optical fibers, remote controls, and biometric authentication. ${ }^{10-13}$ Currently, when high-precision optical communications, medical diagnoses, and environmental measurements are required, high-brightness NIR light and its high-sensitivity detection are indispensable. However, the photodetection and luminescence efficiencies of NIR light are lower than those of visible light. Therefore, a need exists for the development of materials and devices that effectively use NIR light.

The more effective use of light requires the amplification of "invisible light", its conversion into "visible light" or "usable energy," and its subsequent detection. Thus, the purpose of the present research is to create novel material systems that enable the detection and manipulation of invisible light (weak light, polarized light, and NIR light) and to apply them to optoelectronics via the fabrication of organic-inorganic hybrid materials. Inorganic materials (inorganic semiconductors) are basically characterized by high electrical conductivity, high thermal stability, and good durability. In contrast, organic molecular materials have advantages over inorganic materials, including better light absorption, broader material diversity, greater flexibility, and better compatibility with solutionbased manufacturing processes. However, simply combining these materials physically does not result in exploiting their respective functions, but in formation of an inorganic-organic heterointerface with poor functional properties. So, controlling the heterointerfaces in organic-inorganic hybrid structures can lead to the creation of new optical and electrochemical functionalities. In the present study, a new method of chemically bonding organic-inorganic interfaces at the molecular level through complexation has been proposed. At the heterointerface formed by this method, the photoelectric conversion and energy conversion processes caused by light absorption of the materials can be controlled. Here, a new material and device for the ultra-efficient amplification, conversion, and detection of invisible light (weak light, polarized light, and NIR light) at room temperature and pressure, achieved through precise control of the heterointerface in organic-inorganic hybrid structures, is reported.

\section{Photocurrent Amplification and Highly Sensitive Visible- light Detection by Organic-Inorganic Hybrid Interfaces}

To achieve the high signal-to-noise ratio (i.e., high detection sensitivity) required for the detection of weak light, such as in highresolution imaging, optical communications, single-photon counting, and biochemical sensing applications, it is essential to amplify the photocurrent above the theoretical quantum efficiency limit $(100 \%)$. In general, inorganic photodetectors such as Si and GaAs provide a large amplification gain by photoelectron emission ${ }^{1}$ and the avalanche effect; ${ }^{2,3}$ however, these devices require a high external bias $(\sim 100 \mathrm{~V})$ for operation. Alternatively, photocurrent amplification can be achieved via a photomultiplication phenomenon in which trap-assisted charge-tunneling injection at a heterogeneous interface contributes to a large leakage current. Photomultiplication-type detectors have been fabricated using organic materials ${ }^{14,15}$ and hybrid materials such as organic-inorganic hybrid perovskites. ${ }^{16,17}$ In organic photodetectors, large external electron (or hole) injection is induced by the accumulation of photogenerated carriers. However, large charge injection in an organic layer will result in a large dark current, which can lower the sensitivity of the photodetector.

In the present study, we develop a novel photomultiplication-type detector using a metal complex as an interfacial charge-tunneling layer between a perovskite-type absorber and a metal electrode (cathode), ${ }^{18}$ resulting in a detector with highly sensitive and efficient photodetection under a low driving voltage at room temperature. The chemical bonding of an organic semiconductor and a lead halide perovskite via metal ions can form a unique interface that induces photocurrent amplification, where trap-assisted chargetunneling injection at a heterogeneous interface contributes to a large leakage current. Here, this unique interface is shown to detect weak light with more than 2000 times greater photocurrent amplification at very low driving voltages $(<1 \mathrm{~V})$. Reducing the applied voltage enables not only energy-saving operation but also downsizing of the device.

A bilayer photodetector consisting of methylammonium lead iodide $\left(\mathrm{MAPbI}_{3}\right)$ nanoparticles as a light absorber and a molecular layer consisting of an $\mathrm{Eu}^{3+}$ complex with $2,2^{\prime}: 6^{\prime}, 2^{\prime \prime}$-terpyridine (terpy) as a charge-tunneling layer ${ }^{19}$ was constructed on a $180 \mathrm{~nm}$ thick mesoporous $\mathrm{TiO}_{2}\left(\right.$ meso- $\left.\mathrm{TiO}_{2}\right)$ layer coated onto a transparent conducting oxide (TCO) glass substrate (meso- $\mathrm{TiO}_{2} / \mathrm{MAPbI}_{3} / \mathrm{Eu}-$ terpy, Fig. 1a). Nanoparticles of $\mathrm{MAPbI}_{3}(2-3 \mathrm{~nm})$ were obtained by spin-coating low-concentration $(<0.5 \mathrm{M})$ precursor solutions of MAI and $\mathrm{PbI}_{2}$ onto $\mathrm{TiO}_{2}$, followed by an annealing process. The organic insulating molecule (terpy) and perovskite active layer were chemically cross-linked via Eu ions. All these layers were fabricated by solution-coating processes under ambient air. Finally, a Ag counter electrode was formed on top of the bilayer structure by vacuum deposition. To reduce the dark current, the surface of the TCO was coated with a compact thin film of $\mathrm{Eu}_{2} \mathrm{O}_{3}$ as a holeblocking layer.

The device responded to weak visible light with wavelengths longer than $495 \mathrm{~nm}$ and generated a photocurrent (Fig. 1b). The photocurrent amplification factor (or gain value) for low-intensity light at $550 \mathrm{~nm}\left(0.76 \mathrm{~mW} \mathrm{~cm}^{-2}\right)$ reached 2900 , corresponding to an external quantum conversion efficiency (EQE) (i.e., an incidentphoton-to-current conversion efficiency) of $2.9 \times 10^{5} \%$, at an applied voltage of $-0.5 \mathrm{~V}$ (Fig. 1b; this EQE, to the best of our knowledge, is the highest reported thus far for a perovskite-based photodetector. A similar high EQE was also obtained at weak incident power as low as $10 \mu \mathrm{W} \mathrm{cm}{ }^{-2}$. The corresponding photoresponsivity $(R)$, which describes the ability of a photodetector to respond to optical signals, was $1289 \mathrm{AW}^{-1}$. This responsivity obtained for visible-light detection under a low applied bias $(-0.5 \mathrm{~V})$ is superior to that of avalanche photodiodes based on $\mathrm{Si}$ or GaAs, in which a large amplified photocurrent is achieved under an applied strong external electric field $(\sim 100 \mathrm{~V})$. The large photoamplification and large $R$ of the $\mathrm{MAPbI}_{3}$ nanoparticle-based devices with a Eu-terpy complex are largely a consequence of substantial suppression of the dark current by the Eu-terpy complex and the large photoconductivity of $\mathrm{MAPbI}_{3}$, which resulted in a high signal-to-noise ratio of $10^{6}-10^{7}$.

In this device, the thin/molecular layer of the Eu-terpy complex between $\mathrm{MAPbI}_{3}$ and $\mathrm{Ag}$ performs the critical role of suppressing 
(a)
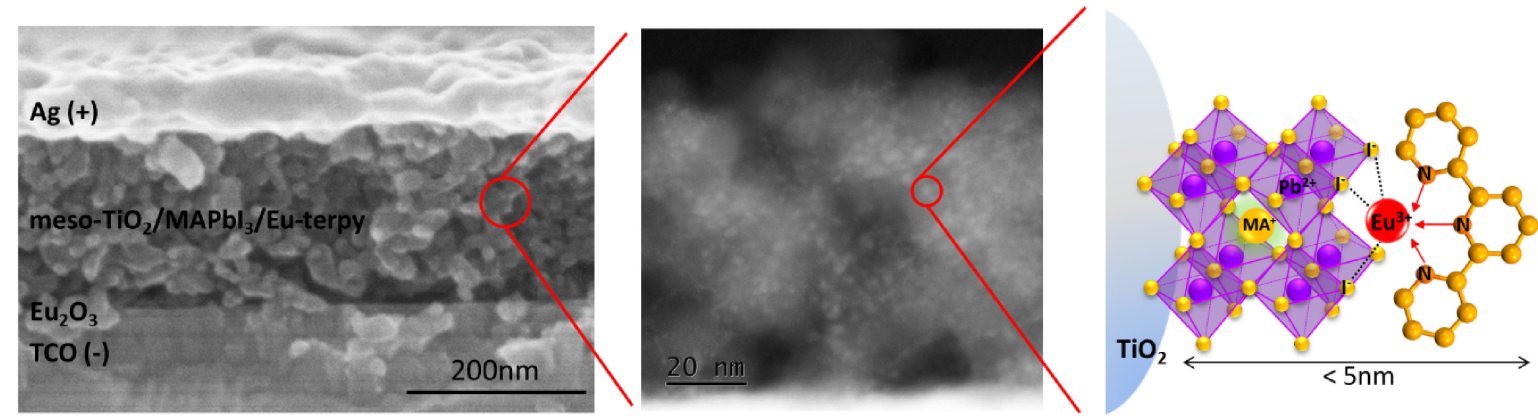

(b)

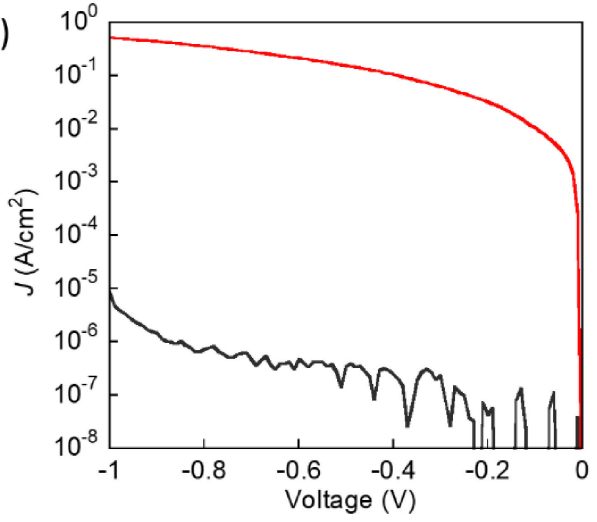

(c)

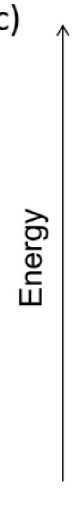

in dark

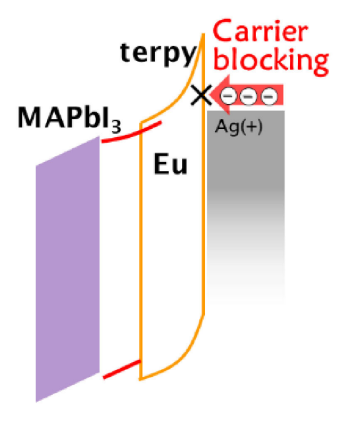

under light

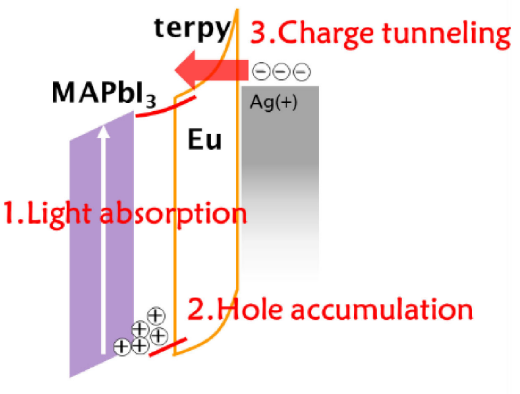

Figure 1. (a) SEM and TEM cross-sectional images of the TCO/Eu $\mathrm{O}_{3} /$ meso- $\mathrm{TiO}_{2} / \mathrm{MAPbI}_{3} / \mathrm{Eu}-\mathrm{terpy} / \mathrm{Ag}$ device and a junction model of the $\mathrm{MAPbI}_{3}$ nanostructure with Eu-terpy complex on meso- $\mathrm{TiO}_{2}$. (b) $J-V$ curves under irradiations of weak visible light with wavelengths over $495 \mathrm{~nm}$ (red line, $0.7 \mathrm{~mW} \mathrm{~cm}^{-2}$ ) and dark current (gray line). (c) Schematic diagram of the photoamplification process of the device, which has the thin/molecular layer of the Eu-terpy complex between $\mathrm{MAPbI}_{3}$ and $\mathrm{Ag}$, under applied reverse bias.

the dark current and, more importantly, allows electron-tunneling injection into $\mathrm{MAPbI}_{3}$ (Fig. 1c). $\mathrm{MAPbI}_{3}$ nanoparticles on a meso$\mathrm{TiO}_{2}$ layer efficiently absorb visible light $(<800 \mathrm{~nm})$. Under photoexcitation of the $\mathrm{MAPbI}_{3}$, electrons transfer to the conduction band of $\mathrm{TiO}_{2}$ and the holes created in the valence band of $\mathrm{MAPbI}_{3}$ accumulate at the interface between the $\mathrm{MAPbI}_{3}$ and the Eu-terpy complex. The accumulated holes under the reverse-bias potential generate a built-in field at the interface, which enables electron tunneling from the electrode (Ag) to the conduction band of the $\mathrm{MAPbI}_{3}$, resulting in amplification of the photocurrent. For photocurrent amplification in this system, the relationship between the positions and energy levels of $\mathrm{TiO}_{2}$ (n-type semiconductor), $\mathrm{MAPbI}_{3}$ (photodetector layer), and the Eu-terpy layer (tunnel injection layer) is important. On the basis of our estimate of the energy levels of each layer from the corresponding photoelectron spectra, ${ }^{20,21}$ we found that the valence band of $\mathrm{MAPbI}_{3}$ is shallower than the highest occupied molecular orbital levels of Eu and terpy, which enables the accumulation of holes induced by light irradiation. This energy relationship leads to a concentration of the electric field at the thin-film layer, which promotes the external large tunneling electron injection from the electrode, resulting in photocurrent amplification exceeding $10^{5} \%$ even at low voltages $(-0.5 \mathrm{~V})$.

\section{Direct Detection of Circular Polarized Light in Organic-} Inorganic Hybrid Thin Films with a One-dimensional (1D) Helical Structure

Polarized light contains useful information that cannot be detected by the human eye. For example, polarized light incident on an object changes into various states through reflection, transmission, and scattering, making it possible to visualize the detailed state of the object's surface, which is difficult to recognize with ordinary light. ${ }^{22-24}$ Circularly polarized light (CPL), in particular, reflects various information about an object, such as the birefringence and stress distribution when the object is bent. Thus, the development of polarization sensors for detecting circularly or linearly polarized light has attracted intensive attention in recent years because such sensors could presumably be used to visualize the birefringence and stress of objects. ${ }^{25-28}$ However, in the structure of existing polarization sensors, a polarizer array is stacked on a photodiode and the polarization direction is separated for each pixel, resulting in reduced detection sensitivity and a lower extinction ratio. In addition, the detection of CPL requires a quarterwavelength plate, which substantially reduces sensitivity; this design has consequently not been implemented in practice.

CPL occurs when an electric field rotates in a circle around the direction of propagation and is referred to as left-handed or righthanded CPL depending on the direction of rotation. Circular dichroism (CD) is the differential absorption between left- and righthanded CPL and is observed in the absorption bands of optically active molecules and chiral secondary structures. The use of materials with such chirality has led to the direct detection of CPL. Yang et al. demonstrated an organic field-effect transistor that detects CPL using an asymmetrically pure, helically shaped chiral semiconducting molecule called helicene. ${ }^{29}$ Schulz et al. detected CPL using an organic field-effect transistor based on enantiopure squaraine derivatives in bulk heterojunction photodiodes. ${ }^{30}$ However, these photodetectors exhibited lower $R$ values $\left(<10 \mathrm{mAW}^{-1}\right)$ than commercially available inorganic photodiodes because of the use of organic molecules with low conductivity; the intrinsically small CD signal ( $\sim 100 \mathrm{mdeg})$ and low polarization discrimination ratio between right- and left-handed CPL detections, $R_{\mathrm{L}} / R_{\mathrm{R}}$, makes their practical use for sensitive CPL detection difficult.

In the present study, to directly detect CPL with a high extinction ratio and sensitivity, a photoconductive crystalline thin film with a 
IL (a)

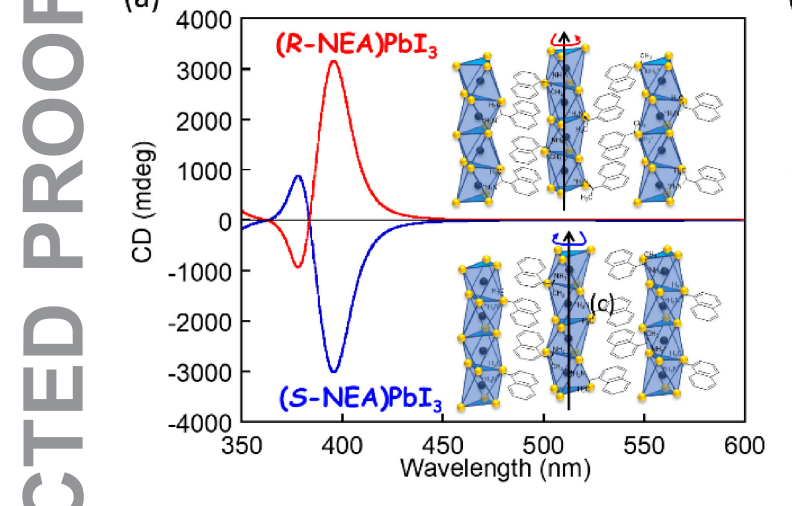

(b)

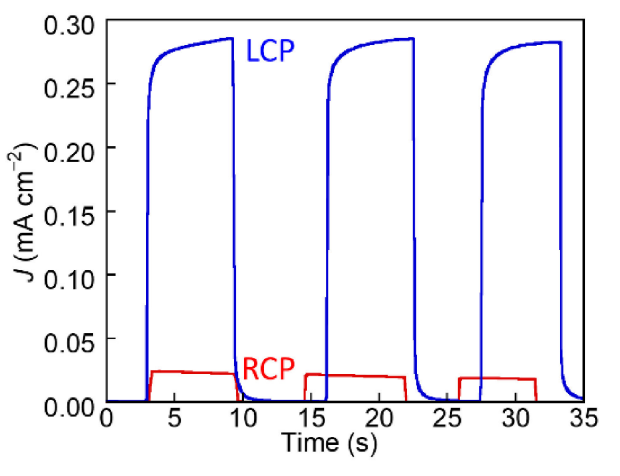

(c)

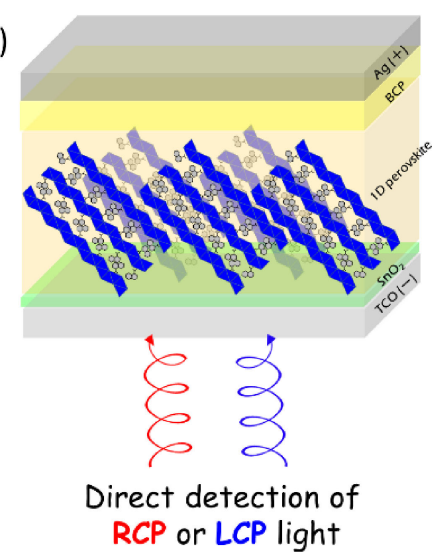

Figure 2. (a) CD spectra of $1 \mathrm{D}$ crystalline thin films (red line, $(R-\mathrm{NEA}) \mathrm{PbI}_{3}$; blue line, $\left.(S-\mathrm{NEA}) \mathrm{PbI}\right)$. (b) Time course of photoresponse $(J-T$ curves) of ( $R$-NEA) $\mathrm{PbI}_{3}$-based device under monochromatic LCP or RCP light irradiation of $1.0 \mathrm{~mW} \mathrm{~cm}^{-2}(395 \mathrm{~nm})$ at an applied voltage of $-0.5 \mathrm{~V}$. (c) Schematic diagram of the helical $1 \mathrm{D}$ perovskite-based photodetector.

chiral structure was constructed by introducing organic chiral molecules into an inorganic layer or chain structure consisting of lead halide perovskites. ${ }^{31}$ The lead halide perovskites generally used in solar cells and similar devices have an achiral structure and therefore cannot discriminate CPL, although they demonstrate high photoelectric conversion performance. Interestingly, we induced large chirality in low-dimensional perovskite thin films by controlling the formation process of the perovskite structure with organic chiral molecules.

$R$-(+)- and $S$-(-)-1-(1-naphtyl)ethylamine ( $R$ - and $S$-NEA ${ }^{+}$, respectively) were used as the organic chiral molecules. The reaction of $\mathrm{PbI}_{2}$ with $R$ - or $S$-NEA ${ }^{+}$yielded low-dimensional chiral crystalline thin films with inorganic layers $\left((R-\text { or } S-N E A)_{2} \mathrm{PbI}_{4}\right.$, two-dimensional (2D)) or chain-like structures ( $R$ - or $S-\mathrm{NEA}) \mathrm{PbI}_{3}$, one-dimensional (1D)) with connected octahedral structures comprising $\left(\mathrm{PbI}_{6}\right)^{4-}$. X-ray diffraction (XRD) measurements and structural analyses based on Rietveld refinements and density functional theory (DFT) calculations ${ }^{32}$ revealed that the layered structure consisted of $2 \mathrm{D}$ sheets with the $\left(\mathrm{PbI}_{6}\right)^{4-}$ octahedra sharing four corners of equatorial halides and $R$ - or $S$-NEA ${ }^{+}$arranged between the sheets. In the case of the chain-like structure, the facesharing $\left(\mathrm{PbI}_{6}\right)^{4-}$ octahedra formed a helical 1D chain (Fig. 2a) in which face-sharing $\left(\mathrm{PbI}_{6}\right)^{4-}$ octahedral chains closely interact with $\mathrm{NEA}^{+}$cations.

The CD spectra of 1D crystalline thin films show opposite positive and negative signals depending on whether the chiral molecules are in the $R$ - or $S$-conformation (Fig. 2a). The results confirm that the chirality was also retained even in the organicinorganic hybrid structure. In the $\mathrm{CD}$ spectrum of the $2 \mathrm{D}$ sheet structure, the signal observed at $\sim 500 \mathrm{~nm}$ is several tens of times stronger than that of common organic chiral molecules. ${ }^{33}$ Surprisingly, the spectrum of the $1 \mathrm{D}$ structure with helical $\left(\mathrm{PbI}_{6}\right)^{4-}$ chains shows intense CD signals greater than $3000 \mathrm{mdeg}$, which is two orders of magnitude greater than the intensity of the CD signals of the $2 \mathrm{D}$ structure and is the highest value reported for a chiral low-dimensional perovskite. These results indicate that the large aromatic naphthyl component of $\mathrm{NEA}^{+}$can induce large helicity in $1 \mathrm{D}\left(\mathrm{PbI}_{6}\right)^{4-}$ chains. The optical absorption properties resulting from the strong interaction between organic chiral molecules and the inorganic crystal arrangements and as a result of strong chirality throughout the film structure can be used to identify CPL components with extremely high sensitivity.

This high CD is directly related to the ability of a photodetector to effectively discriminate between left-handed circularly polarized (LCP) and right-handed circularly polarized (RCP) light. We fabricated a bilayer CPL photodetector using a helical 1D perovskite $\left((R-\right.$ or $\left.S-\mathrm{NEA}) \mathrm{PbI}_{3}\right)$ as a CPL absorber. Figure $2 \mathrm{~b}$ shows the photocurrent responses of the $\left(R\right.$-NEA) $\mathrm{PbI}_{3}$-based device under irradiation with LCP and RCP light. The device shows stronger responses to LCP light than to RCP light. Because the inorganic chain structure consisting of $\mathrm{Pb}$ and $\mathrm{I}$ in this system exhibits high conductivity, the photoelectric conversion efficiency (i.e., the EQE) and sensitivity (i.e., $R$ ) of the device are very high $(87.5 \%$ and $0.28 \mathrm{AW}^{-1}$, respectively) when the device is irradiated with LCP. By contrast, when the device is irradiated with RCP, both the efficiency and sensitivity decrease substantially $(\mathrm{EQE}=3.4 \%$ and $\left.R=0.011 \mathrm{~A} \mathrm{~W}^{-1}\right)$. In the case of the device with $(S-\mathrm{NEA}) \mathrm{PbI}_{3}$ with the opposite helical orientation, the opposite trend was observed with respect to the CPL direction. That is, selective detection of leftor right-handed CPL was achieved using a crystalline thin film with a helical chain structure as a light absorber in the photodetector. The polarization discrimination ratio (ratio between the sensitivity to LCP and that to RCP, $R_{\mathrm{LCP}} / R_{\mathrm{RCP}}$ ), which represents the ability to detect CPL, was 25.4, which is the highest value reported for a device that directly detects CPL. ${ }^{29,30,34,35}$ The high sensitivity to CPL and the high discrimination ratio are attributed to the strong circular polarization absorption and the conduction direction of the helical axis in the 1D perovskite film (Fig. 2c). The performance of the devices reported in the present work exceeds that of previously reported devices for detecting CPL, with or without filters.

\section{Development of Dye-sensitized Up-conversion Nanoparticles and their Application in Near-infrared Photoelectric Conversion Devices}

Compared with visible-light detection, NIR-light detection is difficult to achieve with high sensitivity and low noise. Although numerous approaches to the sensitive detection of NIR light via optimization of NIR-light absorbers have been proposed, our newly proposed approach is to energetically convert weak NIR light into visible light that can be detected with high accuracy using existing technologies and materials. This method not only enables the highly sensitive detection of NIR light as visible light but also makes it possible to achieve an energy conversion efficiency that greatly exceeds the theoretical limit of existing solar cells by converting weak light in the NIR region of sunlight into visible light. In addition, compared with UV and visible light, NIR light exhibits less loss due to light scattering and autofluorescence in biological tissues and can easily penetrate living organisms. The conversion of this highly bio-transparent NIR light into visible light is expected to 
(a)

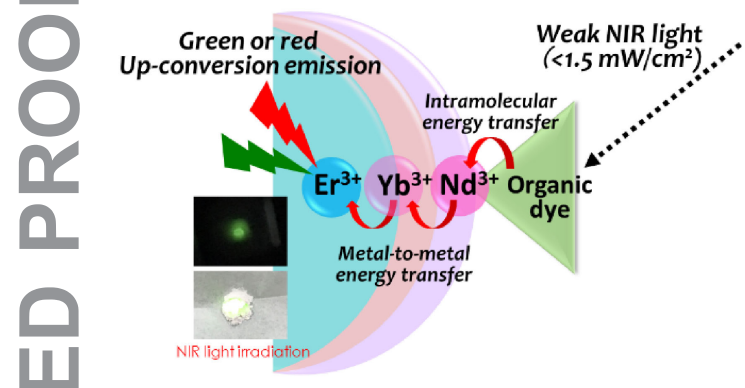

(b)

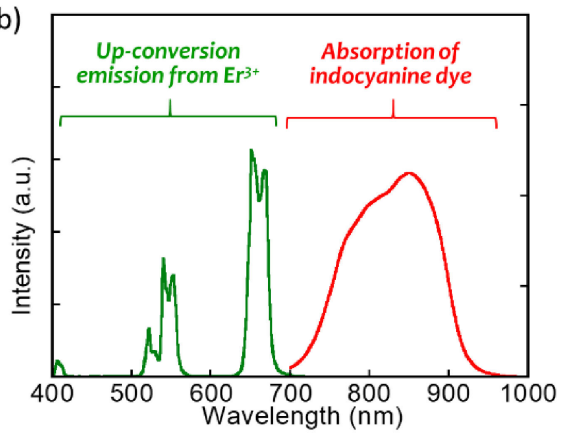

(c)

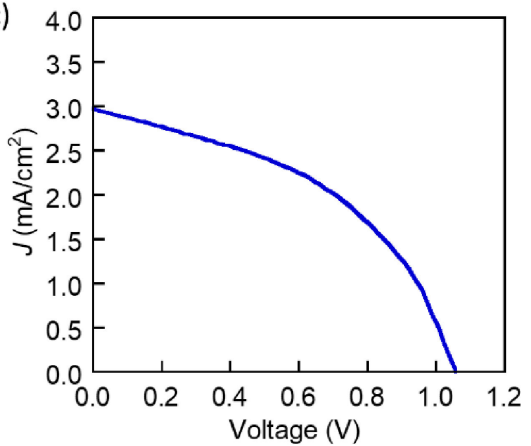

Figure 3. (a) Schematic illustration of a core/shell structured up-conversion nanoparticle coordinated with organic dye and the upconversion luminescence under NIR light irradiation. (b) Absorption and emission spectra of the indocyanine dye-sensitized up-conversion nanoparticles. Up-conversion emission is obtained by excitation with a $\mathrm{CW}$ Xe lamp at $800 \mathrm{~nm}$. (c) $J-V$ curves of $\mathrm{CsPb}_{2} \mathrm{Br}_{-}$based solar cell with up-conversion nanoparticles under irradiation with solar light (1 sun) with wavelengths longer than $750 \mathrm{~nm}$.

open new bio-imaging and optogenetics approaches. Up-conversion materials enable the conversion of NIR light into visible light. In this section, the development of up-conversion materials that convert faint NIR light into visible light and a new NIR-light detection technology based on these materials are introduced.

Up-conversion is a phenomenon in which two or more photons are absorbed sequentially, resulting in the emission of light with a wavelength shorter than the excitation wavelength; up-conversion thus enables the conversion of low-energy light such as NIR light into high-energy light such as visible or UV light. Up-conversion materials that contain lanthanide ions have long been known. For example, NIR light at $980 \mathrm{~nm}$ can be converted into visible light such as blue, green, or red light via energy transfer from $\mathrm{Yb}^{3+}$ ions to $\mathrm{Er}^{3+}$ and $\mathrm{Tm}^{3+}$ ions. ${ }^{36}$ By contrast, up-conversion luminescence is based on electronic transitions between $4 \mathrm{f}$ energy levels of lanthanide ions, which are forbidden transitions, resulting in a remarkably low luminescence efficiency $(\sim 1 \%)$ and a very low light absorption ability (molar absorption coefficient; $\varepsilon=1$ $10 \mathrm{dm}^{3} \mathrm{~mol}^{-1} \mathrm{~cm}^{-1}, 1 / 10000$ of the $\varepsilon$ of a typical organic dye). Thus, the efficient conversion of weak NIR light into visible light using conventional lanthanide-based up-conversion materials is difficult because of their large energetic loss and limited optical functions (e.g., wavelength), although these materials have attractive properties for converting NIR light into visible light. And therefore, the application of up-conversion materials not only to the detection of weak NIR light but also to the highly efficient use of sunlight and to diagnosis and treatment in deep biological tissues faces numerous challenges.

Since lanthanide ions cannot strongly absorb light, they cannot readily detect weak incident NIR light. Therefore, we used an organic dye $\left(\varepsilon=10^{4}-10^{5} \mathrm{dm}^{3} \mathrm{~mol}^{-1} \mathrm{~cm}^{-1}\right)$ as an energy donor for the lanthanide ions; the dye exhibits strong light-absorbing ability in the NIR region, more than 10000 times that of lanthanide ions. When the organic dye absorbs light and its energy matches the energy level of the lanthanide ions, energy transfer occurs with high efficiency. When the organic dye and the lanthanide ion form a complex, the efficiency becomes extremely high and the lanthanide ions show strong luminescence even under weak light irradiation. In the present study, using a new method of applying energy transfer between organic dyes and lanthanide ions to up-conversion, we have achieved up-conversion luminescence even with NIR light, which is weaker than sunlight, by forming complexes between organic dyes with lanthanide ions (hereafter referred to as lanthanide complexes) at the nanoparticle interface. For example, in core-shell-structured oxide nanoparticles containing $\mathrm{Tm}^{3+}$ and $\mathrm{Yb}^{3+}$ with organic dyes (indigo and squaraine dyes), the NIR light absorbed by the organic dyes can be sequentially transferred to $\mathrm{Yb}^{3+}$ and $\mathrm{Tm}^{3+} .37,38$ This system, which utilizes the complexation with organic dyes and energy transfer at the nanoparticle interface, is the first example of a system that demonstrates successful up-conversion emission in the blue region $(475 \mathrm{~nm})$ under NIR-light irradiation with an intensity less than $1 / 10$ th that of sunlight $\left(0.1 \mathrm{~mW} \mathrm{~cm}^{-2},>640 \mathrm{~nm}\right)$. In addition, we have reported systems using indocyanine and phthalocyanine dyes, which exhibit strong absorption in the NIR region (700-900 nm), as organic dyes. For example, in the case of indocyanine dye, $\mathrm{Nd}^{3+}$ with multiple energy levels at $\sim 800 \mathrm{~nm}$ is a suitable acceptor. When the up-conversion nanoparticles, which are fluorides containing $\mathrm{Er}^{3+}$ and $\mathrm{Yb}^{3+}$ coated with $\mathrm{Nd}^{3+}$, are coupled with indocyanine dyes, the energy transfer efficiency from the dyes to the up-conversion nanoparticles is improved and strong upconversion luminescence is attained even under weak excitation light sources (excitation wavelength: $800 \mathrm{~nm}$ ) such as sunlight (Fig. 3a). The up-conversion luminescence efficiency was greater than $5 \%$ (Fig. 3b), which is the highest level of luminescence efficiency reported for lanthanide-ion-based nanoparticles.

Lead halide perovskite compounds (referred to as "perovskites"), which are expected to replace $\mathrm{Si}$ as a next-generation solar cell material, have shown energy conversion efficiencies greater than $20 \% ;{ }^{39}$ however, because of their bandgap, they can only generate electricity from sunlight in the limited wavelength region below $800 \mathrm{~nm}$ (visible light). The ability to generate electricity from light in the NIR region would likely lead to new fourth-generation solar cells with a conversion efficiency that greatly exceeds the theoretical limit of $\sim 30 \%$. In the present study, we have constructed a new system for converting light in the NIR region, which has not previously been used in perovskite solar cells, into visible light and extracting it as electrical energy by incorporating our new upconversion material into the active layer of perovskite solar cells. In the device, the dye-sensitized up-conversion nanoparticles shown in Fig. $3 \mathrm{a}$ are incorporated into the photoactive layer of a perovskite $\left(\mathrm{CsPbI}_{2} \mathrm{Br}\right)$ solar cell. The $\mathrm{CsPb}_{2} \mathrm{Br}$-based solar cell originally absorbs light with a wavelength less than $700 \mathrm{~nm}$ and does not convert light of longer wavelengths. Surprisingly, the introduction of dye-sensitized up-conversion nanoparticles into the $\mathrm{CsPb}_{2} \mathrm{Br}$ photoactive layer enables power generation under irradiation with light with wavelengths longer than $750 \mathrm{~nm}$ (Fig. 3c). Although the low efficiency still poses a challenge (power conversion efficiency; $2.53 \%$ ), this work represents the first observation of power generation from weak NIR light at the level of sunlight using the up-conversion phenomenon.

Another problem with up-conversion nanoparticles containing lanthanide ions is that only $\sim 2 \%$ of the particles can be doped with luminescent species $\left(\mathrm{Er}^{3+}, \mathrm{Tm}^{3+}\right.$, etc. $)$ because of energy deactivation (concentration quenching) at higher dopant concentrations 
resulting in weak luminescence despite the presence of numerous luminescent species. $^{40}$ Here, to suppress the deactivation of luminescence on the nanoparticle surface, the nanoparticles were coated with a low-vibration inorganic medium instead of chain organic molecules typically coated onto the surface of nanoparticles to improve their dispersibility. For example, nanoparticles of $\mathrm{NaErF}_{4}$ coated with chain organic molecules show very weak luminescence despite the presence of $100 \% \mathrm{Er}^{3+}$ (luminescence quantum yield, $\varphi<0.01 \%$ ). We increased the luminescence efficiency to a maximum of $3 \%$ by replacing these chain-like organic molecules with an inorganic layer composed of $\mathrm{CsPbr}_{3}$, which is a perovskite material that has recently attracted much attention for use in nextgeneration solar cells.

We also developed an NIR optical sensor using high-brightness up-conversion nanoparticles with an inorganic $\mathrm{CsPbr}_{3}$ shell layer. The $\mathrm{CsPbBr}_{3}$ shell layer of the nanoparticles has a very high affinity for $\mathrm{CsPbI}_{3}$, the material used in photoactive layer, and forms a highly crystalline and defect-free perovskite thin film. Using the thin film with the up-conversion nanoparticles as the NIR photosensitive layer, we fabricated a stacked type photodetector with the structure. This device responds to weak light with a wavelength of $808 \mathrm{~nm}$. That is, the energy conversion process of the up-conversion nanoparticles from NIR light to visible light can be used to detect NIR light as a photocurrent in the perovskite-based photodetector. Although the response speed requires further improvement, we achieved a high conversion efficiency of $75 \%$ for the detection of weak NIR light.

\section{Fabrication of Heterometallic Hybrid Crystal Thin Films} Converting Single Absorbed Photons into Two Emitted Photons for High-brightness Near-infrared Light-emitting Diodes

NIR light-emitting diodes (LEDs) are useful in a wide range of applications, including night-vision devices, optical communication devices, biomedical imaging instruments, and medical treatments. ${ }^{41-43}$ NIR emitters based on organic compounds and colloidal quantum dots have been widely investigated. ${ }^{44-47}$ However, their poor charge transport ability and low luminescence efficiency limit the EQE of NIR LEDs, which is still far from sufficient for these devices to be used in practical applications. The theoretical limit of luminescence efficiency of general luminescent materials is $100 \%$, where luminescence is achieved by converting a single absorbed photon into a single emitted photon. If the single absorbed photon can be converted into two emitted photons, highly efficient luminescence exceeding $100 \%$ (up to $200 \%$ ) becomes possible. ${ }^{48}$

In the present study, we developed brilliant NIR luminescent materials based on the one-photon-absorption-to-two-photon-emission process, referred to as "quantum-cutting," via energy transfer. The quantum-cutting energy transfer system was composed of an all-inorganic perovskite $\left(\mathrm{CsPbCl}_{3}\right)$ film as an energy donor and ytterbium ions $\left(\mathrm{Yb}^{3+}\right)$ as an acceptor. $\mathrm{Yb}^{3+}$ ions show an NIR emission band at $\sim 1000 \mathrm{~nm}$ assigned to the ${ }^{2} \mathrm{~F}_{5 / 2} \rightarrow{ }^{2} \mathrm{~F}_{7 / 2}$ transition of the inner-shell $4 f$ orbitals, which can be enhanced through energy transfer from suitable energy donors. Here, we achieved high NIR luminesce of $\mathrm{Yb}^{3+}$ sensitized by a $\mathrm{CsPbCl}_{3}$ crystalline film with high charge-carrier mobility and well-balanced charge injection, resulting in $\mathrm{Yb}^{3+}: \mathrm{CsPbCl}_{3}$-based NIR LEDs with a high EQE value. ${ }^{49}$

Figure 4a shows photoluminescence spectra of $\mathrm{Yb}^{3+}: \mathrm{CsPCl}_{3}$ films. The $\mathrm{Yb}^{3+}$-doped $\mathrm{CsPbCl}_{3}$ thin film was fabricated by a multistep solution-process to obtain a 120 -nm-thick highly transparent thin film (93\% transmittance, Fig. $4 \mathrm{a}$ inset). The emission from $\mathrm{CsPbl}_{3}$ observed at $415 \mathrm{~nm}$ was completely quenched by the $\mathrm{Yb}^{3+}$ dopant. The strong emission is observed at $984 \mathrm{~nm}$, which is assigned to the ${ }^{2} \mathrm{~F}_{5 / 2} \rightarrow{ }^{2} \mathrm{~F}_{7 / 2}$ transitions of $\mathrm{Yb}^{3+}$, by excitation of
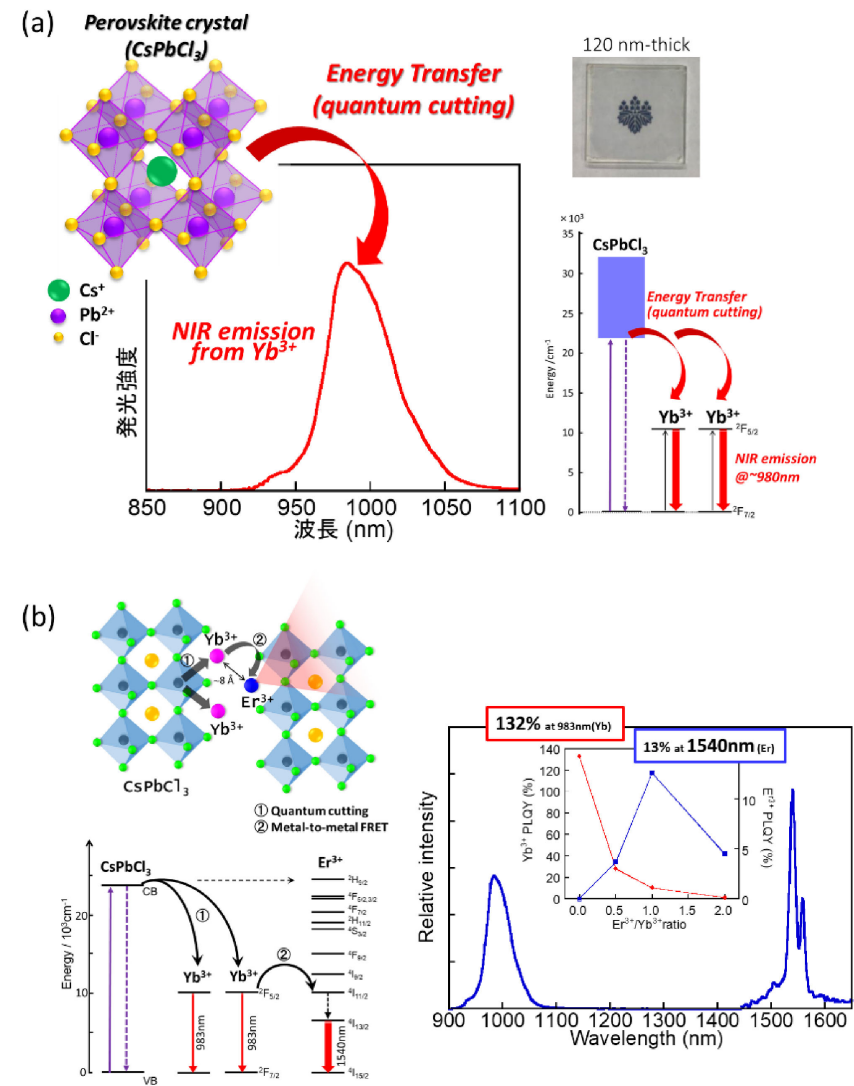

Figure 4. (a) Photoluminescence spectra of $\mathrm{Yb}^{3+}(9.1 \mathrm{~mol} \%)$ : $\mathrm{CsPbCl}_{3}$ film $\left(\lambda_{\mathrm{ex}}=300 \mathrm{~nm}\right)$ and the energy diagram of the quantum cutting from $\mathrm{CsPCl}_{3}$ to $\mathrm{Yb}^{3+}$. (b) Photoluminescence spectra $\left(\lambda_{\mathrm{ex}}=300 \mathrm{~nm}\right)$ of the $\mathrm{Er}^{3+} / \mathrm{Yb}^{3+}$ intercalated $\mathrm{CsPbCl}_{3}$ film with the $\mathrm{Er}^{3+} / \mathrm{Yb}^{3+}$ ratio of $1: 1$ and schematic structural models for energy migration in the $\mathrm{Er}^{3+} / \mathrm{Yb}^{3+}$ intercalated quasi-2D $\mathrm{CsPbCl}_{3}$ layers.

$\mathrm{CsPbCl}_{3}$ at $320 \mathrm{~nm}$. Such strong NIR luminescence was hardly observed by direct excitation of $\mathrm{Yb}^{3+}$ at $980 \mathrm{~nm}$ using a strong diode laser $\left(\sim 430 \mathrm{~W} \mathrm{~cm}^{-2}\right)$. The excitation spectrum monitored at $984 \mathrm{~nm}$ matches the absorption spectrum of $\mathrm{CsPbCl}_{3}$. These results suggest that the NIR luminescence of $\mathrm{Yb}^{3+}$ in $\mathrm{CsPbCl}_{3}$ is effectively enhanced by the energy transfer from $\mathrm{CsPbCl}_{3}$ to $\mathrm{Yb}^{3+}$. The lifetime of this emission from $\mathrm{Yb}^{3+}$ in $\mathrm{CsPbCl}_{3}$ was estimated as a single emission component of $1.68 \mathrm{~ms}$, indicating that $\mathrm{Yb}^{3+}$ is uniformly dispersed in the $\mathrm{CsPbCl}_{3}$ film. The NIR emission intensity increases with increasing $\mathrm{Yb}^{3+}$ concentration to $9.1 \mathrm{~mol} \%$ relative to the $\mathrm{Pb}^{2+}$ concentration. The $\mathrm{Yb}^{3+}: \mathrm{CsPCCl}_{3}$ exhibited the highest photoluminescence quantum yield (PLQY) of $62 \%$.

Recently, we effectively enhanced quantum-cutting energy transfer using a highly orientated crystalline film by fixing $\mathrm{Yb}^{3+}$ ions in the $\mathrm{CsPbCl}_{3}$ lattice by preparing a one-to-one layer arrangement comprising quasi-2D $\mathrm{CsPbCl}_{3}$ perovskite and $\mathrm{Yb}^{3+}$ layers. ${ }^{50}$ The successful layer arrangement resulted in highly sensitized luminescence of $\mathrm{Yb}^{3+}$ by $\mathrm{CsPbCl}_{3}$, with NIR PLQYs exceeding $130 \%$, which we attributed to quantum cutting. In addition, $\mathrm{Er}^{3+}$ luminescence at $1540 \mathrm{~nm}$ was achieved with the introduction of $\mathrm{Er}^{3+}$ into the $\mathrm{Yb}^{3+}$-containing layer, which resulted in intralayer metal-to-metal energy transfer (Förster resonance energy transfer, FRET) from $\mathrm{Yb}^{3+}$ activated by $\mathrm{CsPbCl}_{3}$ via the interlayer quantum-cutting process (Fig. 4b). The PLQY of $\mathrm{Er}^{3+}$ luminescence reached $12.6 \%$, which is the highest value ever reported for $\mathrm{Er}^{3+}$ compounds, resulting in an efficient interlayer quantum cutting process greater than $100 \%$ and in intralayer 
I. (a)

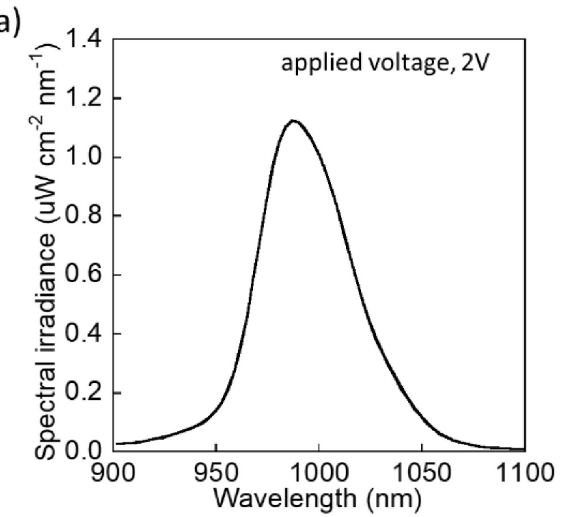

(b)

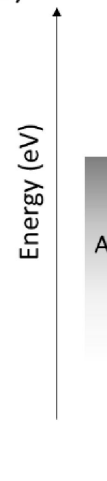

(c)

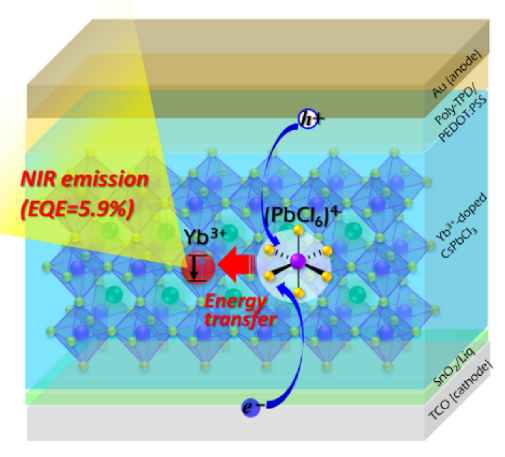

Figure 5. (a) NIR electroluminescence spectrum (applied voltage, $2 \mathrm{~V}$ ) of the $\mathrm{Yb}^{3+}(9.1 \mathrm{~mol} \%): \mathrm{CsPCl}_{3}$-based LED. (b) Energy diagram of the energy transfer type NIR LED. (c) Schematic device structure of the $\mathrm{Yb}^{3+}(9.1 \mathrm{~mol} \%): \mathrm{CsPCl}_{3}$-based LED.

resonance metal-to-metal energy transfer with an efficiency greater than $80 \%$.

Thin-film structures with good carrier-transporting properties retain their luminescence function as light-emitting devices. We therefore fabricated a perovskite NIR LED consisting of $\mathrm{Yb}^{3+}: \mathrm{CsPbCl}_{3}$ film. The $\mathrm{Yb}^{3+}: \mathrm{CsPbCl}_{3}$-based device shows strong NIR electroluminescence at $984 \mathrm{~nm}$ (Fig. 5a) but no luminescence in the visible wavelength region. It suggests that the energy transfer from electrically excited $\mathrm{CsPbCl}_{3}$ to $\mathrm{Yb}^{3+}$ effectively occurs as well as the case of photoexcitation, which is enhanced by effective carrier diffusion of perovskite to an emission center composed of $\mathrm{Yb}^{3+}$ partially replaced in a $\mathrm{CsPbCl}_{3}$ lattice (Fig. 5b). The $\mathrm{Yb}^{3+}: \mathrm{CsPCCl}_{3}$ device exhibits a low turn-on voltage of $\sim 1.6 \mathrm{~V}$, which is attributed to the high-mobility perovskite film and efficient carrier injection from the HTL and ETL, and reaches a high irradiance of $3100 \mu \mathrm{W} \mathrm{cm}{ }^{-1}$ at $3.6 \mathrm{~V}$. The EQE value of the $\mathrm{Yb}^{3+}: \mathrm{CsPCCl}_{3}$ device reaches a maximum of $5.9 \%$ at $0.087 \mathrm{~mA} \mathrm{~cm}^{-2}$, which is the highest EQE reported for a thin-film NIR LED with emission wavelengths greater than $900 \mathrm{~nm}$ (Fig. 5c).

\section{Conclusions}

We have established a unique technology to precisely control the photoelectric conversion and energy conversion processes caused by light absorption in materials by using heterointerfaces in organicinorganic hybrid structures and have achieved the amplification, conversion, and detection of "invisible light" such as weak light, polarized light, and NIR light. The approach of light absorption using heterointerfaces has not been previously reported and is an innovative optical technology for the direct detection and manipulation of light with ultra-high efficiency under normal temperatures and pressures. Materials and elements that can precisely detect and use optical information represent a technology that will revolutionize our understanding of life, medical systems, and environmental and energy issues on the basis of existing principles and optical technologies and are expected to substantially contribute to future advances in science and technology.

\section{Acknowledgments}

The author gratefully thanks Professor Tsutomu Miyasaka from Toin University of Yokohama and Professor Ken-ichi Ueda of the University of Electro-Communications for their generous support and advice in carrying out this research. I also acknowledge Professor Hiroshi Segawa and Professor Takaya Kubo from The University of Tokyo for supporting the scanning electron microscopy and XRD measurements. This work was supported by JST
PRESTO grant no. JPMJPR17P2, JSPS KAKENHI, and grant no. $19 \mathrm{H} 04552$.

\section{Funding}

Precursory Research for Embryonic Science and Technology: JPMJPR17P2

Japan Society for the Promotion of Science: $19 \mathrm{H} 04552$

\section{References}

1. T. Hattori, Y. Kawashima, M. Daikoku, H. Inouye, and H. Nakatsuka, Jpn. J. Appl. Phys., 39, L809 (2000).

2. G. J. Rees and J. P. R. David, J. Phys. D: Appl. Phys., 43, 243001 (2010).

3. S. Kasap, J. A. Rowlands, S. D. Baranovskii, and K. J. Tanioka, Appl. Phys., 96, 2037 (2004)

4. S. Miki, F. Marsili, and A. Casaburi, Supercond. Sci. Technol., 29, 050301 (2016).

5. K. Niwa, T. Numata, K. Hattori, and D. Fukuda, Sci. Rep., 7, 45660 (2017).

6. E. Masvidal-Codina, X. Illa, M. Dasilva, A. B. Calia, T. Dragojević, E. E. VidalRosas, E. Prats-Alfonso, J. Martínez-Aguilar, J. M. De la Cruz, R. GarciaCortadella, P. Godignon, G. Rius, A. Camassa, E. D. Corro, J. Bousquet, C. Hébert, T. Durduran, R. Villa, M. V. Sanchez-Vives, J. A. Garrido, and A. Guimerà-Brunet, Nat. Mater., 18, 280 (2019).

7. R. Florentin, V. Kermene, J. Benoist, A. Desfarges-Berthelemot, D. Pagnoux, A. Barthélémy, and J.-P. Huignard, Light Sci. Appl., 6, e16208 (2017).

8. M. Parniak, M. Dąbrowski, M. Mazelanik, A. Leszczyński, M. Lipka, and W. Wasilewski, Nat. Commun., 8, 2140 (2017).

9. J. B. Haun, N. K. Devaraj, S. A. Hilderbrand, H. Lee, and R. Weissleder, Nat. Nanotechnol., 5, 660 (2010).

10. J. Haglund, F. Jeppsson, E. Melander, A.-M. Pendrill, C. Xie, and K. J. Schönborn, Infrared Phys. Technol., 75, 150 (2016).

11. M. A. Jatoi and N. Kamel, J. Eng., 2014, 426 (2014).

12. G. Tao, H. Ebendorff-Heidepriem, A. M. Stolyarov, S. Danto, J. V. Badding, Y. Fink, J. Ballato, and A. F. Abouraddy, Adv. Opt. Photonics, 7, 379 (2015).

13. D. Tordera, B. Peeters, E. Delvitto, S. Shanmugam, J. Maas, J. Riet, R. Verbeek, R. Laar, T. Bel, G. Haas, L. Ugalde, A. Breemen, I. Katsouras, A. J. Kronemeijer, H. Akkerman, E. Meulenkamp, and G. Gelinck, J. Soc. Inf. Disp., 28, 381 (2020).

14. M. Hiramoto, A. Miki, M. Yoshida, and M. Yokoyama, Appl. Phys. Lett., 81, 1500 (2002).

15. J. Miao and F. Zhang, Laser Photonics Rev., 13, 1800204 (2019).

16. K. Domanski, W. Tress, T. Moehl, M. Saliba, M. K. Nazeeruddin, and M. Grätzel, Adv. Funct. Mater., 25, 6936 (2015).

17. R. Dong, Y. Fang, J. Chae, J. Dai, Z. Xiao, Q. Dong, Y. Yuan, A. Centrone, X. C. Zeng, and J. Huang, Adv. Mater., 27, 1912 (2015).

18. A. Ishii, A. K. Jena, and T. Miyasaka, J. Phys. Chem. Lett., 10, 5935 (2019).

19. A. Ishii, T. Sakai, R. Takahashi, S. Ogata, K. Kondo, T. Kondo, D. Iwasawa, S. Mizushima, K. Yoshihara, and M. Hasegawa, ACS Appl. Mater. Interfaces, 10, 5706 (2018).

20. W. J. Evans, Coord. Chem. Rev, 206-207, 263 (2000).

21. A. Ishii and M. Hasegawa, Chem. Lett., 45, 1265 (2016).

22. R. Wehner and B. Lanfranconi, Nature, 293, 731 (1981).

23. T. W. Cronin, N. Shashar, R. L. Caldwell, J. Marshall, A. G. Cheroske, and T. H. Chiou, Comp. Biol., 43, 549 (2003).

24. A. Basiri, X. Chen, J. Bai, P. Amrollahi, J. Carpenter, Z. Holman, C. Wang, and Y. 
Yao, Light Sci. Appl., 8, 78 (2019).

25. B. Javidi and T. Nomura, Opt. Eng., 39, 2439 (2000).

26. L. Nagdimunov, L. Kolokolova, and D. Mackowski, J. Quant. Spectrosc. Radiat. Transf., 131, 59 (2013)

27. L. Cen, Z. Zhang, J. Zhang, S. Li, Y. Sun, L. Yan, Y. Zhao, and F. Wang, Phys. Rev. A, 96, 053846 (2017)

28. B. Kunnen, C. Macdonald, A. Doronin, S. Jacques, M. Eccles, and I. Meglinski, J. Biophotonics, 8, 317 (2015).

29. Y. Yang, R. C. Da Costa, M. J. Fuchter, and A. J. Campbell, Nat. Photonics, 7, 634 (2013).

30. M. Schulz, F. Balzer, D. Scheunemann, O. Arteaga, A. Lützen, S. C. J. Meskers, and M. Schiek, Adv. Funct. Mater, 29, 1900684 (2019).

31. A. Ishii and T. Miyasaka, Science Adv, 6, eabd3274 (2020).

32. B. Delley, J. Chem. Phys., 113, 7756 (2000).

33. H. Tanaka, Y. Inoue, and T. Mori, ChemPhotoChem, 2, 386 (2018).

34. C. Chen, L. Gao, W. Gao, C. Ge, X. Du, Z. Li, Y. Yang, G. Niu, and J. Tang, Nat. Commun., 10, 1927 (2019).

35. W. Li, Z. J. Coppens, L. V. Besteiro, W. Wang, and A. O. Govorov, Nat. Commun., 6, 8379 (2015)

36. H. Dong, L.-D. Sun, and C.-H. Yan, Chem. Soc. Rev., 44, 1608 (2015).

37. A. Ishii and M. Hasegawa, Sci. Rep., 7, 41446 (2017).
38. A. Ishii, Y. Adachi, A. Hasegawa, M. Komaba, S. Ogata, and M. Hasegawa, Sci. Technol. Adv. Mater, 20, 44 (2019).

39. T. Miyasaka, Bull. Chem. Soc. Jpn., 91, 1058 (2018).

40. W. Wei, Y. Zhang, R. Chen, J. Goggi, N. Ren, L. Huang, K. K. Bhakoo, H. Sun, and T. T. Y. Tan, Chem. Mater, 26, 5183 (2014).

41. V. J. Pansare, S. Hejazi, W. J. Faenza, and R. K. Prud'homme, Chem. Mater, 24, 812 (2012).

42. F. Ding, Y. Zhan, X. Lub, and Y. Sun, Chem. Sci., 9, 4370 (2018).

43. I. L. Medintz, H. T. Uyeda, E. R. Goldman, and H. Mattoussi, Nat. Mater, 4, 435 (2005).

44. A. Zampetti, A. Minotto, and F. Cacialli, Adv. Funct. Mater, 29, 1807623 (2019).

45. D.-H. Kim, A. D'Aléo, X.-K. Chen, A. D. S. Sandanayaka, D. Yao, L. Zhao, T. Komino, E. Zaborova, G. Canard, Y. Tsuchiya, E. Choi, J. W. Wu, F. Fages, J.-L. Brédas, J.-C. Ribierre, and C. Adachi, Nat. Photonics, 12, 98 (2018).

46. K. T. Ly, R.-W. Chen-Cheng, H.-W. Lin, Y.-J. Shiau, S.-H. Liu, P.-T. Chou, C.-S. Tsao, Y.-C. Huang, and Y. Chi, Nat. Photonics, 11, 63 (2017).

47. Y. Shirasaki, G. J. Supran, M. G. Bawendi, and V. Bulović, Nat. Photonics, 7, 13 (2013).

48. D. L. Dexter, Phys. Rev., 108, 630 (1957)

49. A. Ishii and T. Miyasaka, Adv. Sci., 7, 1903142 (2020).

50. A. Ishii and T. Miyasaka, J. Chem. Phys., 153, 194704 (2020). 